\title{
Short-Term Drug-Drug Interaction between Sildenafil and Bosentan under Long-Term Use in Patients with Pulmonary Arterial Hypertension
}

\author{
Sachiko Miyakawa ${ }^{1 *}$, Keiichi Odagiri ${ }^{1}$, Naoki Inui ${ }^{1}$, Akio Hakamata ${ }^{1}$, Takahiro Goto ${ }^{2}$, \\ Shimako Tanaka ${ }^{3}$, Shinya Uchida ${ }^{3}$, Noriyuki Namiki ${ }^{3}$, Hiroshi Watanabe ${ }^{1}$ \\ ${ }^{1}$ Department of Clinical Pharmacology and Therapeutics, Hamamatsu University School of Medicine, Hamamatsu, Japan; ${ }^{2}$ Depart- \\ ment of Drug Evaluation and Informatics, School of Pharmaceutical Sciences, University of Shizuoka, Shizuoka, Japan; ${ }^{3}$ Department \\ of Pharmacy Practice and Science, School of Pharmaceutical Science, University of Shizuoka, Shizuoka, Japan. \\ Email: "s_miya@hama-med.ac.jp
}

Received August $23^{\text {rd }}, 2013$; revised September $25^{\text {th }}, 2013$; accepted October $9^{\text {th }}, 2013$

Copyright (C) 2013 Sachiko Miyakawa et al. This is an open access article distributed under the Creative Commons Attribution License, which permits unrestricted use, distribution, and reproduction in any medium, provided the original work is properly cited.

\begin{abstract}
Sildenafil and bosentan are often co-administered for pulmonary arterial hypertension (PAH) treatment. The plasma concentration of sildenafil can be decreased by half if co-administered with bosentan. Many patients take these agents simultaneously in the morning and the evening. The aim of this study was to examine the pharmacokinetics of sildenafil which was interfered with bosentan administration to ascertain whether these agents should be given concomitantly or separately. A two-way crossover study was conducted in 6 PAH patients with combination therapy of sildenafil and bosentan. Participants underwent the sequence of treatment phases: phase S (sildenafil administered $3 \mathrm{~h}$ before bosen$\tan$ ); phase B (bosentan administered $3 \mathrm{~h}$ before sildenafil); and phase C (administered concomitantly). Blood samples were collected on the last day of each phase. There was no significant difference in maximum plasma concentration or area under the plasma concentration-time curve $\left(\mathrm{AUC}_{0-8}\right)$ between phase $\mathrm{C}$ and phase $\mathrm{S}(95.5 \pm 24.8$ vs. $72.9 \pm 40.9$ ( $\mathrm{p}=$ $0.07), 209.7 \pm 81.8$ vs. $180.2 \pm 126.4(\mathrm{p}=0.24)$, respectively) or between phases $\mathrm{C}$ and $\mathrm{B}(87.8 \pm 42.0 \mathrm{vs} .99 .6 \pm 33.9$ ( $\mathrm{p}$ $=0.59), 197.2 \pm 88.2$ vs. $240.7 \pm 121.8(\mathrm{p}=0.19)$, respectively) $(\mathrm{ng} / \mathrm{mL}$, mean \pm standard deviation). Large intra- and inter-individual variability in sildenafil concentration was noted. The timing of administration of sildenafil and bosentan does not significantly influence the plasma concentration of sildenafil. Physicians do not need to be overly concerned about the timing of administration of these drugs to maximize the sildenafil concentration.
\end{abstract}

Keywords: Drug-Drug Interaction; Pulmonary Arterial Hypertension; Sildenafil; Bosentan; Pharmacokinetics

\section{Introduction}

Pulmonary arterial hypertension $(\mathrm{PAH})$ is a progressive and proliferative disease of the small pulmonary vasculature. PAH is characterized by vasoconstriction, thrombosis in situ, and vascular remodeling [1,2]. PAH leads to a progressive increase in pulmonary arterial pressure (PAP) and pulmonary vascular resistance to provoke right ventricular dysfunction and, ultimately, death. In the 1980 s, PAH was thought to be a deadly disease because the median survival after the diagnosis was 2.8 years [3].

Since 1990, several PAH-specific drugs have been approved for the indication of this orphan disease and survival does seem to have improved in the modern era

"Corresponding author.
$[4,5]$. Currently, various treatments that focus on symptom relief and improving the prognosis are available: prostanoids, phosphodiesterase-5 (PDE-5) inhibitors, and endothelin receptor antagonists [1]. Sildenafil (a selective inhibitor of cyclic guanosine monophosphate) and bosentan (an antagonist of endothelin-A and -B receptors) are drugs used in the treatment of PAH. Sildenafil improves exercise capacity and reduces PAP [6]. Bosentan reduces PAP and improves exercise capacity as well as functional class in patients with $\mathrm{PAH}[7,8]$.

A combination of a PDE-5 inhibitor and endothelin-1 receptor antagonist is standard therapy for advanced PAH [9]. Sildenafil and bosentan are often co-administered but crucial drug-drug interactions between these agents have been reported. Studies have shown the 
plasma concentration of sildenafil to be decreased by $50 \%$ in the area under the plasma concentration-time curve (AUC) when co-administered chronically with bosentan in PAH patients [10], whereas the efficacy of sildenafil is dose dependent [11].

Sildenafil and bosentan are recommended to be administered at 8 -h intervals (i.e., three times daily (TID)) and 12-h intervals (i.e., twice daily (BID)), respectively, but not a few patients take these drugs simultaneously in the morning and evening. The difference in these timings of administration might have an appreciable influence on drug-drug interactions. We wanted to know if the pharmacokinetics of sildenafil was interfered with bosentan administration. In this way, physicians would then know whether these agents could be given concomitantly or separately.

\section{Methods}

This study protocol complied with the Declaration of Helsinki and was approved by the Research Review Board of Hamamatsu University School of Medicine (Hamamatsu, Japan). Written informed consent was provided by all participants. The study was registered at the UMIN Clinical Trials Registry (UMIN000002566).

\subsection{Study Design}

This was a single-center, open-label, randomized, twoway crossover, drug-drug interaction study conducted from January 2008 to March 2009. Enrolled participants were randomly allocated to either treatment pattern 1 or 2 . Pattern 1 began with phase $\mathrm{C}$ and phase $\mathrm{S}$ followed by phase $\mathrm{C}$ and phase $\mathrm{B}$, while pattern 2 started with phase $\mathrm{C}$ and phase $\mathrm{B}$ followed by phase $\mathrm{C}$ and phase $\mathrm{S}$ (Figure 1). Phase $\mathrm{C}$ (concomitant administration) comprised sildenafil (Revatio ${ }^{\mathrm{TM}}$; Pfizer, Tokyo, Japan; $20 \mathrm{mg}$; TID) at $07.00 \mathrm{~h}, 13.00 \mathrm{~h}$, and $19.00 \mathrm{~h}$, while bosentan (Tracleer $^{\mathrm{TM}}$; Actelion Pharmaceuticals, Tokyo, Japan; $62.5 \mathrm{mg}$; BID) was administered at $07.00 \mathrm{~h}$ and $19.00 \mathrm{~h}$. Phase S (sildenafil-first) comprised sildenafil $(20 \mathrm{mg}$; TID) at $07.00 \mathrm{~h}, 13.00 \mathrm{~h}$, and $19.00 \mathrm{~h}$, and bosentan $(62.5$ $\mathrm{mg}$; BID) at $10.00 \mathrm{~h}$ and $22.00 \mathrm{~h}$. Phase B (bosentan-first) comprised sildenafil (20 mg; TID) at $10.00 \mathrm{~h}, 16.00 \mathrm{~h}$, and $22.00 \mathrm{~h}$, and bosentan (62.5 mg; BID) at $07.00 \mathrm{~h}$ and 19.00 h. Participants followed the instruction of drug administration at home during the treatment phases.

We also undertook a preliminary study of sildenafil monotherapy for two participants (participant numbers 1 and 2) to confirm chronic drug-drug interactions between sildenafil and bosentan.

\subsection{Inclusion and Exclusion Criteria}

Eligible participants were patients with PAH who received

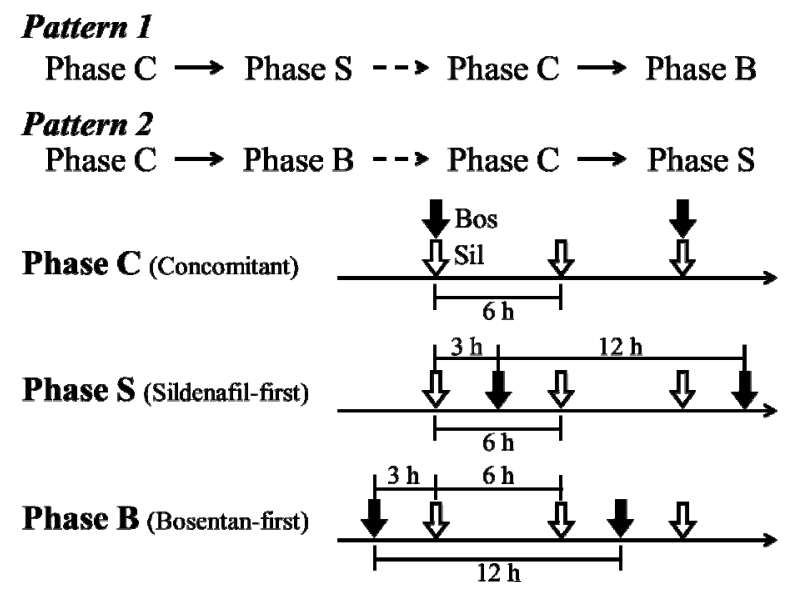

Figure 1. Details of protocol patterns and phases. Phase $C$ comprises sildenafil (20 mg; TID) at $07.00,13.00$, and 19.00 $\mathrm{h}$, whereas bosentan (62.5 $\mathrm{mg}$; BID) is at $07.00 \mathrm{~h}$ and 19.00 h. Sildenafil and bosentan are administered simultaneously at $07.00 \mathrm{~h}$ and $19.00 \mathrm{~h}$. Phase $\mathrm{S}$ comprises sildenafil $(20 \mathrm{mg}$; TID) at $07.00,13.00$, and $19.00 \mathrm{~h}$, whereas bosentan $(62.5$ mg; BID) is at $10.00 \mathrm{~h}$ and $22.00 \mathrm{~h}$. Sildenafil is administered $3 \mathrm{~h}$ before bosentan administration. Phase $\mathrm{B}$ comprises sildenafil (20 mg; TID) at 10.00, 16.00, and 22.00 h, whereas bosentan (62.5 mg; BID) is at $07.00 \mathrm{~h}$ and 19.00 h. Bosentan is administered $3 \mathrm{~h}$ before sildenafil administration. Sil (blank arrows): sildenafil, Bos (bold arrows): bosentan.

combination therapy of sildenafil (20 mg; TID) and bosentan (62.5 mg; BID) for $>1$ year. The exclusion criteria were poor health status, apparent adverse effects of sildenafil or bosentan, and difficulties in receiving continuous administration of these drugs.

\subsection{Pharmacokinetic and Safety Assessments}

On the last day of each treatment phase, participants visited our institution for blood sampling. Study drugs were administered in the morning to participants in the fasted state, with breakfast served $2 \mathrm{~h}$ thereafter. After the baseline blood collection, participants were given sildenafil $(20 \mathrm{mg})$ and/or bosentan $(62.5 \mathrm{mg})$ according to the protocol of each treatment phase. Blood samples for the measurement of sildenafil were collected $0.5,1$, $1.5,2,3,4,6$, and $8 \mathrm{~h}$ after sildenafil administration. The schedule of blood sampling is shown in Table 1. On the day of blood sampling, the second aliquot of sildenafil of the day was administrated after the last blood sample was collected ( $8 \mathrm{~h}$ later), which matched the recommended regimen of drug administration and was fully tolerable to PAH patients. Participants were confined to the study center until the final blood sample was collected.

The plasma concentration of sildenafil was determined by liquid chromatography/mass spectrometry. Briefly, $100 \mu \mathrm{L}$ of diazepam $(1 \mu \mathrm{g} / \mathrm{mL})$ as an internal standard 
Table 1. Timetable of blood sampling day.

\begin{tabular}{cccccc}
\hline \multicolumn{5}{c}{ Phase C } \\
\hline$h^{a}$ & Time & $\begin{array}{c}\text { Blood } \\
\text { sampling }\end{array}$ & Sildenafil & Bosentan & Meal \\
\hline 0.0 & 07.00 & & $*$ & $*$ & \\
0.5 & 07.30 & $*$ & & & \\
1.0 & 08.00 & $*$ & & & \\
1.5 & 08.30 & $*$ & & & \\
2.0 & 09.00 & $*$ & & \\
3.0 & 10.00 & $*$ & & \\
4.0 & 11.00 & $*$ & & \\
5.0 & 12.00 & & & \\
6.0 & 13.00 & $*$ & & \\
8.0 & 15.00 & $*$ & & \\
\hline
\end{tabular}

\begin{tabular}{cccccc}
\hline \multicolumn{5}{c}{ Phase S } \\
\hline$h$ & Time & $\begin{array}{c}\text { Blood } \\
\text { sampling }\end{array}$ & Sildenafil & Bosentan & Meal \\
\hline 0.0 & 07.00 & & $*$ & & \\
0.5 & 07.30 & $*$ & & & \\
1.0 & 08.00 & $*$ & & & \\
1.5 & 08.30 & $*$ & & & \\
2.0 & 09.00 & $*$ & & & \\
3.0 & 10.00 & $*$ & & & \\
4.0 & 11.00 & $*$ & & & \\
5.0 & 12.00 & & & \\
6.0 & 13.00 & $*$ & & \\
8.0 & 15.00 & $*$ & & \\
\hline
\end{tabular}

\begin{tabular}{cccccc}
\hline \multicolumn{5}{c}{ Phase B } \\
\hline$h$ & Time & $\begin{array}{c}\text { Blood } \\
\text { sampling }\end{array}$ & Sildenafil & Bosentan & Meal \\
\hline- & 07.00 & & & $*$ & \\
0.0 & 10.00 & & $*$ & & \\
0.5 & 10.30 & $*$ & & & \\
1.0 & 11.00 & $*$ & & & \\
1.5 & 11.30 & $*$ & & \\
2.0 & 12.00 & $*$ & & \\
3.0 & 13.00 & $*$ & & \\
4.0 & 14.00 & $*$ & & \\
6.0 & 16.00 & $*$ & & \\
8.0 & 18.00 & $*$ & & \\
\hline
\end{tabular}

a-hours from sildenafil administration.

was added to the plasma sample (500 $\mu \mathrm{L})$. Cold acetonitrile $(500 \mu \mathrm{L})$ was added to the sample and vortex mixed to enable deproteination. After centrifugation of $3000 \mathrm{rpm}$ for $10 \mathrm{~min}$ at $4^{\circ} \mathrm{C}$, the supernatant fluid was diluted with water $(1.5 \mathrm{~mL})$ and applied to an OASIS
HLB extraction cartridge (Waters, Milford, MA, USA). The cartridge was washed with $5 \%$ methanol in water $(1.0 \mathrm{~mL})$ and eluted with acetonitrile $(1.0 \mathrm{~mL})$. The eluate was evaporated under a stream of nitrogen gas at $40^{\circ} \mathrm{C}$. Micromass ZQ Mass Spectrometer (Waters, Milford, MA, USA) was operated in positive ion mode at $\mathrm{m} / \mathrm{z}$ 475. The limit of quantification was $1 \mathrm{ng} / \mathrm{mL}$, and the intra-assay coefficient of variation was $<6.40 \%$.

The pharmacokinetic parameters for sildenafil were estimated by non-compartmental analyses from the concentration-time profile in plasma. The terminal elimination half-life $\left(t_{1 / 2}\right)$ during the log-linear terminal phase was calculated from the elimination rate constant determined by linear regression analyses. The $\mathrm{AUC}_{0-8}$ was calculated using the trapezoidal rule for the observed values and subsequent extrapolation to $8 \mathrm{~h}$. The oral clearance was calculated as dose/AUC ${ }_{8-\infty}$. The maximum plasma concentration $\left(\mathrm{C}_{\max }\right)$ and the time of maximum concentration $\left(\mathrm{T}_{\max }\right)$ were estimated directly from the observed plasma concentration-time data.

Safety was evaluated by the findings of physical examination and vital signs (systemic blood pressure and pulse rate) at each blood sampling. Biochemical and hematological assessments (blood cell count and serum levels of creatinine, aspartate aminotransferase, and alanine aminotransferase) were undertaken at the first and last visit to our institution.

\subsection{Statistical Analyses}

Data are the mean \pm standard deviation (SD) of the indicated numbers. A paired t-test was used to assess differences in the pharmacokinetic parameters of sildenafil between phases $\mathrm{C}$ and $\mathrm{S}$ as well as phase $\mathrm{B}$. A p-value of $<0.05$ was considered significant. Computations were done using GraphPad Prism version 5.0 (GraphPad Software, La Jolla, CA, USA).

\section{Results}

\subsection{Participant Characteristics}

Six participants with PAH (two men; age, 37 - 66 years) in World Health Organization (WHO) functional class II-III were enrolled. Four participants were classified as having idiopathic PAH and two subjects with associated PAH. The mean height $( \pm \mathrm{SD})$ was $158.5 \pm 9.9 \mathrm{~cm}$, and the mean weight $( \pm \mathrm{SD})$ was $46.4 \pm 7.5 \mathrm{~kg}$. Some participants had complications: interstitial pneumonia (n $=2)$, diabetes mellitus $(n=2)$, scleroderma $(n=1)$, systemic lupus erythematosus $(\mathrm{n}=1)$, polymyositis $(\mathrm{n}=$ $1)$, hyperthyroidism $(\mathrm{n}=1)$, osteoporosis $(\mathrm{n}=1)$, and gout $(\mathrm{n}=1)$. Two participants had no other disease than PAH. 
Participants were taking the following co-administered medications: furosemide $(n=5)$, spironolactone $(n=4)$, beraprost $(\mathrm{n}=4)$, prednisolone $(\mathrm{n}=3)$, rebamipide $(\mathrm{n}=$ $3)$, warfarin $(n=2)$, alendronate $(n=2)$, aspirin $(n=1)$, roxatidine $(\mathrm{n}=1)$, lansoprazole $(\mathrm{n}=1)$, sarpogrelate $(\mathrm{n}=$ 1), allopurinol $(\mathrm{n}=1)$, tocopherol $(\mathrm{n}=1)$, zolpidem $(\mathrm{n}=$ $1)$, paroxetine $(n=1)$, and potassium citrate $(n=1)$. No changes to these medications were made during the study. All participants completed the protocol without worsening of clinical assessments (including routine blood tests).

\subsection{Pharmacokinetics}

First, we undertook a preliminary study to confirm the potential drug-drug interaction of bosentan on the pharmacokinetics of sildenafil. The sildenafil concentration-time curves of sildenafil monotherapy and concomitant administration of sildenafil and bosentan are shown in Figure 2. Compared with sildenafil monotherapy, the plasma concentrations of sildenafil were lower if administered concomitantly with bosentan $(62.5 \mathrm{mg})$. This effect resulted in a decrease in $\mathrm{C}_{\max }$ of $40.8 \%$ for participant 1 and $54.3 \%$ for participant 2, and a decrease in $\mathrm{AUC}_{0-8}$ of $54.0 \%$ for participant 1 and $53.8 \%$ for participant 2.

Next, we evaluated the inter- and intra-individual variability of the sildenafil concentration during concomitant administration with bosentan. The sildenafil concentration-time curves for each participant in phase $\mathrm{C}$ are shown in Figure 3. Inter- and intra-individual variations were considerable. The same dose of sildenafil $(20 \mathrm{mg})$ resulted in an $\mathrm{AUC}_{0-8}$ of $79.7-370.6 \mathrm{~h} \cdot \mathrm{ng} / \mathrm{mL}$ among the six participants in phase $\mathrm{C}$. The intraindividual difference was $1.0-2.3$ times when comparing the two values in phase $\mathrm{C}$ for each participant.

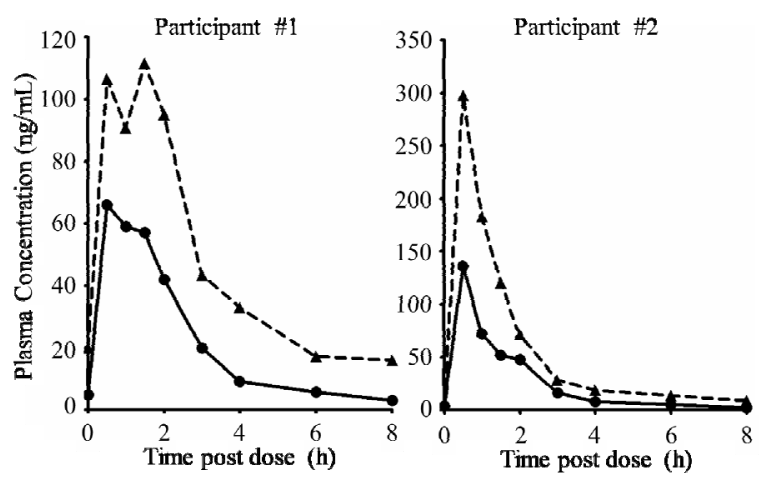

Figure 2. Change in plasma concentration of sildenafil in participants 1 and 2. Sildenafil monotherapy vs. sildenafil plus bosentan. Plasma concentration-time curve for participants 1 and 2 in the preliminary study after administration of sildenafil (20 mg; TID) alone (dotted line) and with bosentan (62.5 mg; BID) (solid line).

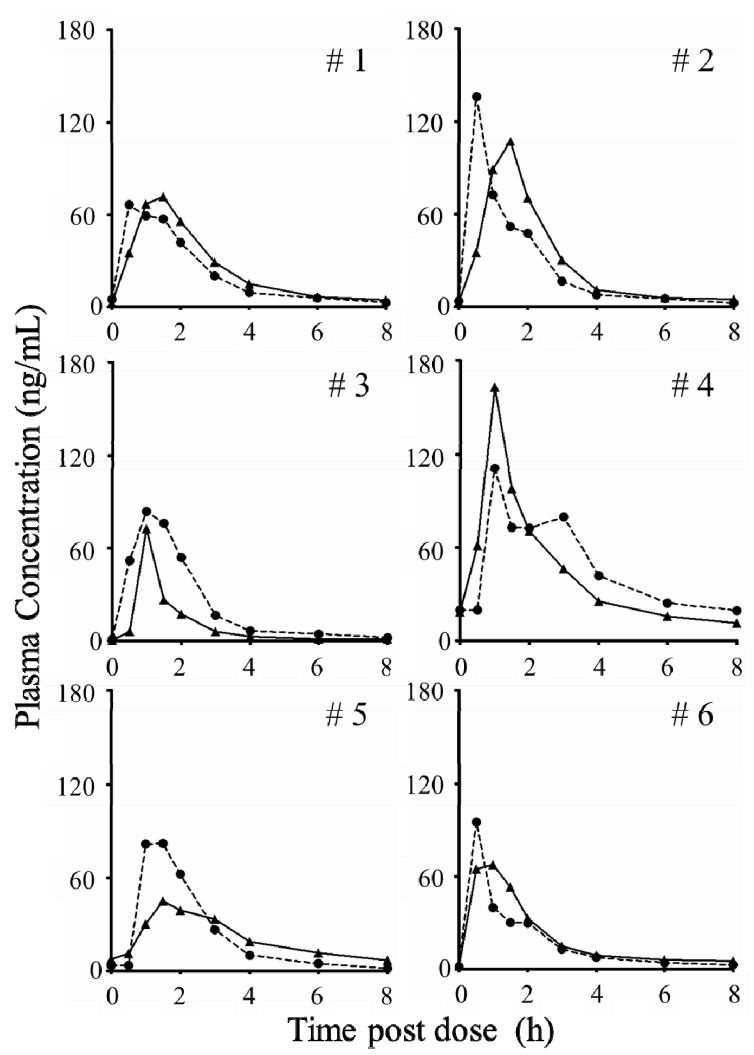

Figure 3. Inter- and intra-individual variability of sildenafil concentration. Sildenafil concentration-time curves in phase C for participants 1 - 6. As the participants underwent phase $C$ twice, both solid line and dotted line show the sildenafil concentration of phase $\mathrm{C}$ at different time.

Finally, we investigated how the timing of administration influenced the sildenafil concentration. The sildenafil concentration-time curves comparing phase $\mathrm{C}$ with phase $\mathrm{S}$ are shown in Figure 4. The pharmaco-kinetics of sildenafil in phase $\mathrm{S}$ was similar to that in phase C. A significant difference in $\mathrm{AUC}_{0-8}$ or $\mathrm{C}_{\max }$ was not shown (Figure 5). The sildenafil concentration-time curves comparing phase $\mathrm{C}$ with phase $\mathrm{B}$ are shown in Figure 6: a significant difference in $\mathrm{AUC}_{0-8}$ or $\mathrm{C}_{\max }$ was not shown (Figure 7). A summary of these pharmaco-kinetic parameters is shown in Table 2.

\section{Discussion}

We investigated the potential interaction between bosentan and sildenafil in relation to the timing of administration of these drugs. We revealed that the sildenafil concentration was not substantially influenced whether bosentan was administered concomitantly or administered separately by $3 \mathrm{~h}$. Furthermore, even in the treatment phase $\mathrm{C}$ (administered concomitantly) there was a considerably large intra-individual variability in sildenafil concentration. 


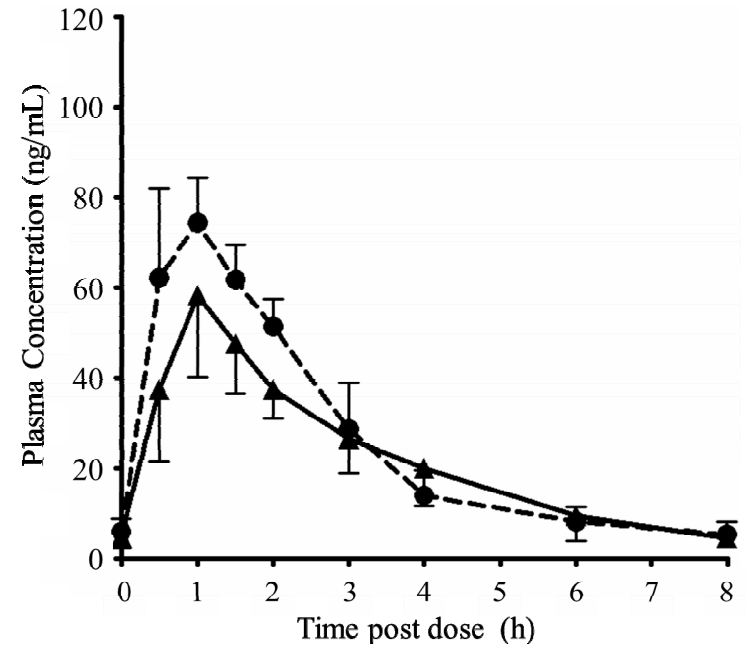

Figure 4. Sildenafil concentration: phase $\mathrm{C}$ vs. phase S. Mean sildenafil concentration-time curves in phase $C$ (dotted line) and phase S (solid line).
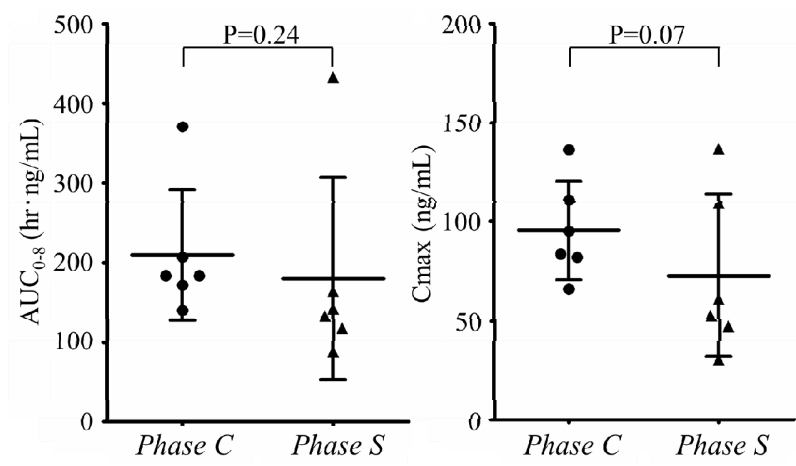

Figure 5. $A_{U C} C_{0-8}$ and $C_{\text {max }}$ for sildenafil: phase $C$ vs. phase $\mathrm{S}$. Mean $\mathrm{AUC}_{0-8}$ (left) and mean $\mathrm{C}_{\max }$ (right) in phase $\mathrm{C}$ and phase S.

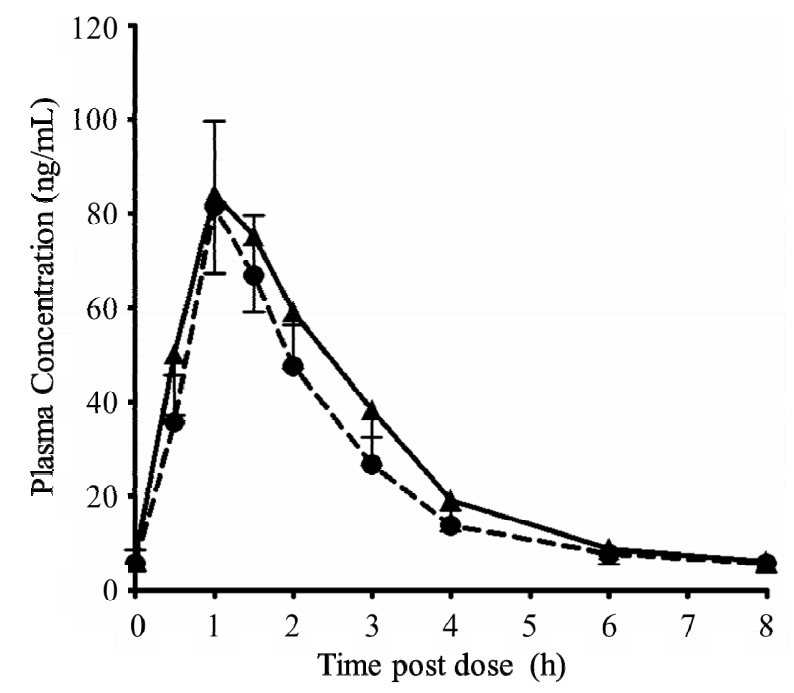

Figure 6. Sildenafil concentration: phase C vs. phase B. Mean sildenafil concentration-time curves in phase $C$ (dotted line) and phase B (solid line).
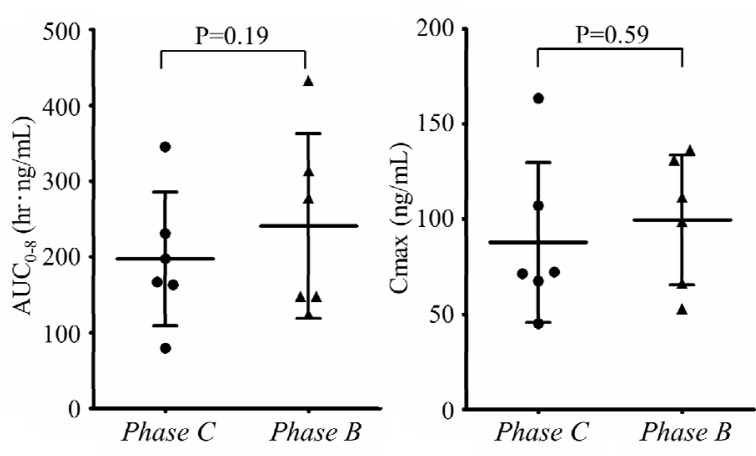

Figure 7. $\mathrm{AUC}_{0-8}$ and $\mathrm{C}_{\max }$ for sildenafil: phase $\mathrm{C}$ vs. phase B. Mean $A C_{0-8}$ (left) and mean $C_{\text {max }}$ (right) in phase $\mathrm{C}$ and phase B.

Table 2. Pharmacokinetic parameters of sildenafil at each phase.

\begin{tabular}{cccc}
\hline & Phase C & Phase $S$ & P value \\
\hline $\mathrm{Cmax}^{\mathrm{a}}(\mathrm{ng} / \mathrm{mL})$ & $95.5(24.8)$ & $72.9(40.9)$ & 0.07 \\
$\mathrm{Tmax}^{\mathrm{b}}(\mathrm{h})$ & $0.8(0.4)$ & $1.3(0.6)$ & 0.14 \\
$\mathrm{t} 1 / 2^{\mathrm{c}}(\mathrm{h})$ & $2.2(0.6)$ & $1.9(0.6)$ & 0.32 \\
$\mathrm{AUC}_{0-8}{ }^{\mathrm{d}}(\mathrm{h} \cdot \mathrm{ng} / \mathrm{mL})$ & $209.7(81.8)$ & $180.2(126.4)$ & 0.24 \\
\hline \multicolumn{5}{c}{} \\
\hline & Phase C & Phase B & P value \\
\hline $\mathrm{Cmax}^{\mathrm{a}}(\mathrm{ng} / \mathrm{mL})$ & $87.8(42.0)$ & $99.6(33.9)$ & 0.59 \\
$\mathrm{Tmax}^{\mathrm{b}}(\mathrm{h})$ & $1.3(0.3)$ & $1.1(0.4)$ & 0.17 \\
$\mathrm{t} 1 / 2^{\mathrm{c}}(\mathrm{h})$ & $2.6(1.6)$ & $1.8(0.4)$ & 0.23 \\
$\mathrm{AUC}_{0-8}{ }^{\mathrm{d}}(\mathrm{h} \cdot \mathrm{ng} / \mathrm{mL})$ & $197.2(88.2)$ & $240.7(121.8)$ & 0.19 \\
\hline
\end{tabular}

Each value is expressed by mean (SD) except $P$ value. $n=6$. a-maximum concentration, b-time of maximum concentration, c-t1/2 terminal elimination half-life, d-area under the plasma concentration-time curve from $0 \mathrm{~h}$ to $8 \mathrm{~h}$ after sildenafil administration.

Several clinical studies have shown significant drugdrug interactions between sildenafil and bosentan. It has been reported that bosentan (125 mg; BID) decreased the $\mathrm{C}_{\max }$ of sildenafil by $55.4 \%$ and $\mathrm{AUC}_{\text {tau }}$ by $62.6 \%$ in healthy volunteers [12]. The mechanism of action of this drug interaction has been rationalized. That is to say, bosentan induces expression of cytochrome P450 3A4 (CYP3A4) in the liver and intestinal wall via activation of the pregnane $\mathrm{X}$ receptor [13], by which sildenafil is meta- bolized mainly into the less active metabolite UK-103, $320[14,15]$. In the present study, we confirmed that the steady-state of bosentan $(62.5 \mathrm{mg}$; BID) also reduced the $\mathrm{C}_{\max }$ of sildenafil by $40 \%-55 \%$ and $\mathrm{AUC}_{0-8}$ by $54 \%$ (Figure 2). This finding suggests that even a lower dose of bosentan can cause drug-drug interactions through CYP3A4 metabolism.

A significant difference in the plasma level of sildenafil was not found between concomitant administration (phase $\mathrm{C}$ ) and time-separated administration of bosentan (phase $\mathrm{S}$ or phase B) but an interesting de- 
creasing trend in sildenafil concentration in phase $\mathrm{S}$ (sildenafil-first) rather than phase $\mathrm{C}$ was noted (Figure 4). This phenomenon is explicable through a short-term drug-drug interaction between sildenafil and bosentan with respect to CYP3A4 metabolism. Given that CYP3A4 activity is already elevated by chronic use of bosentan, sildenafil administered alone should be metabolized readily by a larger amount of activated CYP3A4. Bosentan is not only an inducer but also a substrate of CYP3A4 [15]. Therefore, bosentan administered concomitantly can be a competitive inhibitor against sildenafil to CYP3A4. Consequently, more of the sildenafil can escape from the enzymatic metabolism upon concomitant administration with bosentan. In addition, in vitro studies have suggested that bosentan has an inhibitory effect on CYP3A4. However, the therapeutic dose (125 mg; BID) yields a maximum free-plasma concentration of $\approx 0.08$ $\mu \mathrm{mol} / \mathrm{L}$, which is $\geq 800$-fold lower than the required plasma level for efficient inhibition [16]. Accordingly, the inhibitory effect of bosentan against CYP3A4 in the clinical setting does not have to be considered.

Orally administered sildenafil is rapidly absorbed (92\%) [17]. The detailed mechanism of action of drug transporters such as human organic anion transporting polypeptides (OATPs) and the intestinal efflux pump MDR1 (P-glycoprotein, ABCB1) engaging with sildenafil has yet to be defined. With respect to the drugdrug interaction with bosentan, these transporters will probably not alter the pharmacokinetics of sildenafil because 1) the peak plasma concentration of bosentan at the recommended dose (125 mg; BID) is $\geq 50$-fold lower than that required for efficient inhibition of uptake of OATPs [18] and 2) bosentan is neither a substrate nor an inhibitor of MDR1 [19]. There are very few studies on the distribution or excretion of sildenafil, especially those relevant to drug-drug interactions. Taking into consideration that $\approx 80 \%$ of the administered oral dose of sildenafil is excreted as metabolites predominantly in the feces [20], excretion of sildenafil in urine does not seem to play an important part in drug-drug interactions.

Large variability in sildenafil concentrations within the same individual was noted. Extrinsic conditions were almost identical in the two phase $\mathrm{C}$ regimens: drug administration, duration of fasting, observed general conditions, and the environment within the institution. Therefore, this result suggests intrinsic changes affecting the plasma concentration of sildenafil (e.g., slight changes in the intestinal mucosal membrane due to local edema). This large variability could have nullified the subtle changes in sildenafil concentration, necessitating a greater number of participants to detect significant differences between treatment phases.

The present study had several limitations. First, the small number of participants must be considered. Not only the intra-individual differences described above, but also inter-individual differences in sildenafil concentrations presented substantial barriers. One patient showed a distinguishingly higher sildenafil concentration than the other subjects. Second, CYP3A4 activity in vivo could not be measured directly because CYP3A4 activity is determined by phenotype. Typically, steady-state concentrations of CYP3A4 are identified by the plasmaconcentration ratio of the substrate and its metabolite formed by the enzyme. This measurement requires frequent blood sampling throughout the day. Therefore, to identify the rapid change in CYP3A4 activity in vivo is extremely difficult. Third, the present study was focused on the plasma concentration of sildenafil only. Further studies focusing on the plasma concentration of bosentan are needed to clarify the drug-drug interaction in both directions.

Hence, if physicians tell patients to take sildenafil before taking bosentan, a decrease in sildenafil concentration may result. Concomitant or bosentan-first administration could maintain the plasma level of sildenafil.

In conclusion, differences in the timings of administration of sildenafil and bosentan did not influence the plasma concentration of sildenafil. Physicians do not need to be overly concerned about the timing of administration of these drugs (concomitantly or separately) to maximize the sildenafil concentration. The short-term drug-drug interaction between these agents is limited to large intra-individual variability.

\section{Acknowledgements}

The authors thank the patients for their participation in this study.

\section{Conflict of Interest}

\subsection{Funding}

Funding: This research received no specific grant from any funding agency in the public, commercial, or noncommercial sectors.

\subsection{Competing Interests}

Hiroshi Watanabe has received research funding from the Ministry of Health, Labour and Welfare of Japan, Teika, Takeda, Mochida, Pfizer, Asteras, and Daiichi Sankyo, and lecture fees from Pfizer, Acterion, Novartis, Daiich Sankyo, GlaxoSmithKline and Nihon Shinyaku. Uchida Shinya and Noriyuki Namiki have received research funding from Astellas, Takeda, Otsuka, Towa, and Kissei Pharmaceutical Co., Ltd. 


\section{REFERENCES}

[1] M. Humbert, O. Sitbon and G. Simonneau, "Treatment of Pulmonary Arterial Hypertension," The New England Journal of Medicine, Vol. 351, No. 14, 2004, pp. 14251436. http://dx.doi.org/10.1056/NEJMra040291

[2] J. R. Runo and J. E. Loyd, "Primary Pulmonary Hypertension," The Lancet, Vol. 361, No. 9368, 2003, pp. 1533-1544. http://dx.doi.org/10.1016/S0140-6736(03)13167-4

[3] G. E. D’Alonzo, R. J. Barst, S. M. Ayres, E. H. Bergofsky, B. H. Brundage, K. M. Detre, et al., "Survival in Patients with Primary Pulmonary Hypertension. Results from a National Prospective Registry," Annals of International Medicine, Vol. 115, No. 5, 1991, pp. 343-349. http://dx.doi.org/10.7326/0003-4819-115-5-343

[4] R. L. Benza, D. P. Miller, M. Gomberg-Maitland, R. P. Frantz, A. J. Foreman, C. S. Coffey, et al., "Predicting Survival in Pulmonary Arterial Hypertension: Insights from the Registry to Evaluate Early and Long-Term Pulmonary Arterial Hypertension Disease Management (Reveal)," Circulation, Vol. 122, No. 2, 2010, pp. 164-172. http://dx.doi.org/10.1161/CIRCULATIONAHA.109.8981 $\underline{22}$

[5] M. Humbert, O. Sitbon, A. Chaouat, M. Bertocchi, G. Habib, V. Gressin, et al., "Survival in Patients with Idiopathic, Familial, and Anorexigen-Associated Pulmonary Arterial Hypertension in the Modern Management Era," Circulation, Vol. 122, No. 2, 2010, pp. 156-163.

http://dx.doi.org/10.1161/CIRCULATIONAHA.109.9118 $\underline{18}$

[6] G. W. Mikhail, S. K. Prasad, W. Li, P. Rogers, A. H. Chester, S. Bayne, et al., "Clinical and Haemodynamic Effects of Sildenafil in Pulmonary Hypertension: Acute and Mid-Term Effects," European Heart Journal, Vol. 25, No. 5, 2004, pp. 431-436.

http://dx.doi.org/10.1016/j.ehj.2004.01.013

[7] R. N. Channick, G. Simonneau, O. Sitbon, I. M. Robbins, A. Frost, V. F. Tapson, et al., "Effects of the Dual Endothelin-Receptor Antagonist Bosentan in Patients with Pulmonary Hypertension: A Randomised Placebo-Controlled Study," The Lancet, Vol. 358, No. 9288, 2001, pp. 11191123.

http://dx.doi.org/10.1016/S0140-6736(01)06250-X

[8] L. J. Rubin, D. B. Badesch, R. J. Barst, N. Galie, C. M. Black, A. Keogh, et al., "Bosentan Therapy for Pulmonary Arterial Hypertension," The New England Journal of Medicine, Vol. 346, No. 12, 2002, pp. 896-903. http://dx.doi.org/10.1056/NEJMoa012212

[9] H. A. Ghofrani, O. Distler, F. Gerhardt, M. Gorenflo, E. Grunig, W. E. Haefeli, et al., "Treatment of Pulmonary Arterial Hypertension (PAH): Updated Recommendations of the Cologne Consensus Conference 2011," International Journal of Cardiology, Vol. 154, Suppl. 1, 2011, pp. S20S33. http://dx.doi.org/10.1016/S0167-5273(11)70490-9

[10] G. A. Paul, J. S. Gibbs, A. R. Boobis, A. Abbas and M. R. Wilkins, "Bosentan Decreases the Plasma Concentration of Sildenafil When Coprescribed in Pulmonary Hypertension," British Journal of Clinical Pharmacology, Vol. 60, No. 1, 2005, pp. 107-112.

\section{http://dx.doi.org/10.1111/j.1365-2125.2005.02383.x}

[11] N. Galie, H. A. Ghofrani, A. Torbicki, R. J. Barst, L. J. Rubin, D. Badesch, et al., "Sildenafil Citrate Therapy for Pulmonary Arterial Hypertension," The New England Journal of Medicine, Vol. 353, No. 20, 2005, pp. 21482157. http://dx.doi.org/10.1056/NEJMoa050010

[12] G. Burgess, H. Hoogkamer, L. Collings and J. Dingemanse, "Mutual Pharmacokinetic Interactions between Steady-State Bosentan and Sildenafil," European Journal of Clinical Pharmacology, Vol. 64, No. 1, 2008, pp. 43-50. http://dx.doi.org/10.1007/s00228-007-0408-Z

[13] P. L. van Giersbergen, C. Gnerre, A. Treiber, J. Dingemanse and U. A. Meyer, "Bosentan, a Dual Endothelin Receptor Antagonist, Activates the Pregnane X Nuclear Receptor," European Journal of Pharmacology, Vol. 450, No. 2, 2002, pp. 115-121. http://dx.doi.org/10.1016/S0014-2999(02)02075-7

[14] R. Hyland, E. G. Roe, B. C. Jones and D. A. Smith, "Identification of the Cytochrome P450 Enzymes Involved in the N-Demethylation of Sildenafil," British Journal of Clinical Pharmacology, Vol. 51, No. 3, 2001, pp. 239-248. http://dx.doi.org/10.1046/j.1365-2125.2001.00318.x

[15] J. Dingemanse and P. L. van Giersbergen, "Clinical Pharmacology of Bosentan, a Dual Endothelin Receptor Antagonist," Clinical Pharmacokinetics, Vol. 43, No. 15, 2004, pp. 1089-1115. http://dx.doi.org/10.2165/00003088-200443150-00003

[16] A. Viger-Chougnet, C. Funk and M. Pantze, "Drug-Drug Interactions with Bosentan (Ro 47-0203): In Vitro Studies of the Inhibition Potential of Bosentan on the Main Human Cytochrome P450 Isoenzymes," 1997.

http://www.fda.gov/ohrms/dockets/ac/01/briefing/3775b2 _08_biopharm.doc

[17] G. J. Muirhead, D. J. Rance, D. K. Walker and P. Wastall, "Comparative Human Pharmacokinetics and Metabolism of Single-Dose Oral and Intravenous Sildenafil," British Journal of Clinical Pharmacology, Vol. 53, Suppl. 1, 2002, pp. 13S-20S.

http://dx.doi.org/10.1046/j.06-5251.2001.00028.x

[18] A. Treiber, R. Schneiter, S. Hausler and B. Stieger, "Bosentan Is a Substrate of Human $\mathrm{OATP}_{1} \mathrm{~B}_{1}$ and $\mathrm{OATP}_{1} \mathrm{~B}_{3}$ : Inhibition of Hepatic Uptake as the Common Mechanism of Its Interactions with Cyclosporin A, Rifampicin, and Sildenafil," Drug Metabolism and Disposition, Vol. 35, No. 8, 2007, pp. 1400-1407. http://dx.doi.org/10.1124/dmd.106.013615

[19] A. Treiber, R. Schneiter, S. Delahaye and M. Clozel, "Inhibition of Organic Anion Transporting PolypeptideMediated Hepatic Uptake Is the Major Determinant in the Pharmacokinetic Interaction between Bosentan and Cyclosporin A in the Rat," The Journal of Pharmacology and Experimental Therapeutics, Vol. 308, No. 3, 2004, pp. 1121-1129. http://dx.doi.org/10.1124/jpet.103.061614

[20] Pfizer, "Revatio (Sildenafil) Tablets," 1998. http://labeling.pfizer.com/showlabeling.aspx?id=645 